\title{
FEELINGS, THOUGHTS AND EXPERIENCES OF HEALTHCARE PROFESSIONALS WHO RECOVERED AFTER BEING DIAGNOSED WITH COVID-19, A PHENOMENOLOGICAL STUDY
}

\author{
Sadiye Ozcan ${ }^{1} \&$ Nurcan Kirca ${ }^{2}$ \\ ${ }^{1}$ Yalova University Faculty of Health Sciences, Department of Obstetrics \& Gynecological Nursing, Yalova, Turkey \\ ${ }^{2}$ Akdeniz University Faculty of Nursing, Department of Obstetrics \& Gynecological Nursing, Antalya, Turkey \\ received: 30.6.2021; \\ revised: 25.9.2021; \\ accepted: 29.9.2021
}

\section{SUMMARY}

Background: It is very important to protect the physical, mental and social health of healthcare workers who are at risk, are faced with a difficult pandemic process, and have been infected with the disease. In previous studies, the thoughts of healthcare professionals who gave care to coronavirus patients were investigated. The present study is the first study in which experiences and thoughts of healthcare professionals who had coronavirus disease were investigated, and unlike other studies, data were collected through one-to-one, face-to-face interviews. This study was conducted to determine the feelings, thoughts and experiences of healthcare professionals who recovered after being diagnosed with COVID-19.

Subjects and methods: This qualitative study was designed based on Husserlian's phenomenological approach. The participants were selected from healthcare professionals receiving COVID-19 treatment through the purposive sampling method. The interviews were held between September 2020 and February 2021. Colaizzi's qualitative analysis method was used.

Results: After the analysis of the interviews, three themes and seven sub-themes emerged. The theme "fear" has three subthemes: "fear of the unknown", "fear of being infected again" and "fear of transmitting the disease". The theme "social isolation" has two sub-themes: "Unhappiness" and "stigma". Finally, the theme "lack of motivation" has two sub-themes: "excessive workload" and "Inadequate number of employees".

Conclusions: In order to help healthcare workers manage these psychosocial problems they experience, managers should make appropriate planning and should give them psychosocial support. It is recommended that the criteria regarding healthcare professionals' starting work again after they survive the COVID-19 should be reviewed and revised. In particular, healthcare workers who survive illness but cannot fully recover should be evaluated physically and psychologically before going back to work.

Key words: Colaizzi's qualitative analysis - healthcare professionals - phenomenological study - COVID-19

$$
* * * * *
$$

\section{INTRODUCTION}

Coronavirus disease is spreading rapidly around the world. By May 10, 2021, there were 157,897,763 confirmed cases of COVID-19 and 3,287,082 deaths worldwide (World Health Organization 2021). In Turkey, the Ministry of Health allowed the release of coronavirus reports after December 16, 2020. Turkey is experiencing the worst and most difficult times of the pandemic. In Turkey, from the beginning of the pandemic until May 30,2021 , the total number of cases was 5,242,911, and the number of deaths was 47,405 (Republic of Turkey Ministry of Health 2021). The rapidly spreading epidemic particularly causes the health system to have a hard time in Turkey. As the epidemic progressed rapidly throughout Turkey, the hospitals started to reach their full capacities, and the number of healthcare workers who got the disease increased with each passing day. By May 31, 2020, the number of healthcare workers who lost their lives from the disease was 434 (Republic of Turkey Ministry of Health 2021). In every country, health professionals play a central and critical role in providing healthcare to people. Their health and safety is crucial not only for the provision of safe and uninterrupted patient care, but also for the control of any outbreak (Chang et al. 2020; Q. Liu et al. 2020).
Healthcare professionals make great efforts not only to protect themselves from the disease but also to provide the best care for their patients during the pandemic (Gupta \& Sahoo 2020; Lai et al. 2020). Because healthcare professionals who are struggling with this disease at the forefront are faced with an unprecedented situation, they often work beyond their capacity and in unfamiliar environments and may suffer from serious physical and mental health problems (De Kock et al. 2021; Greenberg et al. 2020). It has been observed that two situations come to the fore, which causes health workers to suffer from these problems. One of them is the news on the social media about the pandemic leaving the impression that "no one is safe". The other one is the risk of COVID-19 transmission due to uncertainties about the amount and quality of personal protective equipment (Bahar et al. 2020; De Kock et al. 2021). In addition, healthcare professionals do not know how to provide care for patients with severe conditions with insufficient equipment (De Kock et al. 2021). Healthcare workers faced with such situations are at a great risk of depression, helplessness, hopelessness, feelings of anxiety and panic, intense fears, loneliness, irritability, intolerance, outbursts of anger, reluctance, concentration difficulty, extreme mental fatigue, and sleep and appetite problems (S. Liu et al. 2020; Zhang et al. 2020). The incidence of these mental health 
problems in healthcare workers was determined as $23 \%$ (Jansson \& Rello 2020). It is reported that healthcare professionals may suffer from mental health problems, which may adversely affect the quality of the patient care they give (Nguyen et al. 2020). In addition to all these, fear of being infected with coronavirus can increase the problems healthcare professionals already experience. Their team members' keeping their distance from them and excluding them, and damaged professional reputation can further affect healthcare workers who were infected with COVID-19 and were then recovered (Shanafelt et al. 2020).

It is very important to protect the physical, mental and social health of healthcare workers who are at risk, are faced with a difficult pandemic process, and have been infected with the disease (Bahar et al. 2020). In a country where healthcare workers are not healthy enough, it is very difficult for people to recover easily and receive ideal health care. Moreover, the number of healthcare workers infected with coronavirus is increasing day by day in our country, Turkey. It is very important to identify the feelings, thoughts and experiences of healthcare professionals in order for them to best fight with this pandemic. If healthcare professionals' feelings, thoughts and problems are not understood well enough during the pandemic, their problems cannot be solved and the quality of the service they provide cannot be improved. To the best of our knowledge, there is no study in which the feelings, thoughts and experiences of healthcare professionals who have previously been infected with coronavirus and been recovered are investigated. In previous studies, the thoughts of healthcare professionals who gave care to coronavirus patients were investigated (Galehdar et al. 2020; S. Liu et al. 2020; Sun et al. 2020). Previous studies conducted on contagious infections showed that high levels of anxiety and stress lower health workers' morale, increase absenteeism, and reduce job satisfaction and quality of patient care (Brooks et al. 2011; Imai et al. 2010; Ives et al. 2009). The present study is the first study in which experiences and thoughts of healthcare professionals who had coronavirus disease were investigated, and unlike other studies, data were collected through one-to-one, face-toface interviews. Therefore, the present study was conducted to investigate the feelings, thoughts and experiences of healthcare professionals who were infected with coronavirus and had great difficulties.

\section{SUBJECTS AND METHODS}

\section{Design}

This Husserlian phenomenological qualitative study (Matua \& Van Der Wal 2015) was conducted with healthcare professionals receiving COVID-19 treatment in Turkey. The main event in phenomenology is how the subject experiences events. The phenomenological method is done to clearly reveal the experiences of the subjects regarding the phenomenon in the universe where the study is conducted.

\section{Setting and Participants}

Healthcare professionals working in a training and research hospital in the western part of Turkey constituted the population of the study. The participants were selected from healthcare professionals receiving COVID-19 treatment through the purposive sampling method. The inclusion criteria are as follows: receiving COVID-19 treatment and willing to participate in the study. The sample of the study consisted of 13 participants. The exclusion criterion is being a health professional not diagnosed with COVID-19. Healthcare professionals are asked to have a PCR test 7 days after they are diagnosed with COVID-19, and those whose two consecutive PCR tests are negative performed 24 hours apart, to return to work on the 11th day. The interviews were conducted within 15 days immediately after two consecutive negative PCR tests. It took 1 month for one of the participants to receive two consecutive negative PCR test results.

\section{Data Collection}

The data were collected by means of individual indepth interviews with the participation of both researchers between September 2020 and February 2021 using the personal information form and a semi structured interview guide. The data were collected by the third researcher using the individual, face-to-face interview method. The interviews were conducted by the researcher at the times determined together with the participants. The researcher first contacted the participants, and then interviewed them, and then reached the health professionals from the shift schedules. Before the participants were interviewed, they were informed about what the purpose of the study was and how it would be conducted. Then, after their verbal and written consent was obtained, in-depth individual interviews were conducted with a semi-structured interview form. The interviews were held in the meeting or nurse rooms of the relevant clinics of the hospital. During the in-depth interviews conducted with the participants in a quiet room, the participants were asked to turn off their phones. Data collection was halted based on the saturation of data (i.e. receiving no new information, receiving repeated information, and when no further coding is available). The interview guide consists of four main open-ended questions about the experiences of healthcare professionals. The interviews were held in a quiet area during break hours in clinics where healthcare professionals work. Research questions are below:

- What do you think the corona virus is? How would you define the corona virus? What would you like to say about the corona virus?

- Could you tell us have you given care to a patient during an epidemic or to a patient diagnosed with COVID-19 or at risk of COVID-19 during this pandemic? 
- Could you tell us what you thought and how you felt when you were diagnosed with corona virus?

- Could you tell us about your experiences before and after you were diagnosed with COVID-19?

To reduce the participants' concerns during in-depth interviews, we allowed time for them to express their feelings, as well as to remain silent. We also engaged with participants sensitively, respecting periods of silence and focusing on experiences and their readiness to continue with the interviews. In order to analyze the obtained data well and to use the time efficiently, the data were recorded on a voice recorder. Written consent was obtained from all the participants to record the interviews. Feedback was given to the participants about their answers. The duration of interviews varied between 45 and 60 minutes depending on the responses given by the health professionals and the effects of the disease on them. Transcription of each interview took an average of 5 hours.

\section{Data Analysis}

The Nvivo 10 package program was used to analyze the data. The interviews audio-recorded by the researcher were transferred to the form on the computer. The study data obtained through interviews were analyzed using the content analysis method. The content analysis requires in-depth analysis of the data collected and allows uncovering of the previously unclear themes and dimensions. The basic process in the content analysis is to gather similar data within the framework of certain concepts and themes, and to organize and interpret them so that the reader can easily understand.

In the present study, after the individual interviews were completed, the audio recordings were listened to by the researchers repeatedly and were transcribed verbatim on the computer. The transcriptions were read several times, and the same, similar and different expressions were grouped. The statements grouped were reanalyzed and those repeated most were determined. Then the main themes and sub-themes of the study were determined. The two researchers analyzed the data by discussing, agreeing and making decisions. Three themes and seven sub-themes were determined in the content analysis. In the data assessment, Colaizzi's content analysis method was used to extract significant statements. The contents of the interviews were recorded with the Microsoft Office program and the data obtained were analyzed in seven steps: (1) Transcribing all the participants' descriptions, (2) Extracting significant statements, (3) Creating formulated meanings, (4) Aggregating formulated meanings into theme clusters, (5) Developing an exhaustive description, (6) Identifying the fundamental structure of the phenomenon, (7) Returning to participants for validation (Edward \& Welch 2011).

\section{Rigor of the Study}

During the analysis of the data after the interviews, the description of the participants was taken into consideration. Apart from the researchers, coding was performed by two independent experts and these codings were matched with the themes created by the researchers. Since there was no subjective relationship between the researchers and the participants, the data collection processes were not affected by personal relationships.

\section{Ethical considerations}

Ethical approval to perform the study was obtained from the University of Health Sciences Ethics Committee (No: 2011-KAEK-25 2020/08-09, Date: 05 August 2020). Written and verbal consent of the participants was obtained with the informed consent forms. The participants were informed that their identities would be kept confidential, and code numbers were used throughout the text (Participant 1: P1).

\section{RESULTS}

The mean age of the participating healthcare professionals was 30.23 \pm 8.00 (Min: 22; Max: 49) years. The majority of the participants were nurses and single. Sociodemographic data of the participants are shown in Table 1. In the present study, the feelings, thoughts and experiences of healthcare professionals who recovered after being diagnosed with COVID-19 were investigated. The recovery process of the participants from the COVID-19 disease varies according to the symptoms of the disease. Those who survived the disease with mild symptoms recovered in an average of 11 days, and those with severe symptoms recovered in an average of 24 days. From the qualitative analysis, three main themes emerged with seven subthemes (refers to Table 2).

\section{Theme 1: Fear}

The subthemes of the "fear" theme were as follows: "fear of the unknown", "fear of being infected again" and "fear of transmitting the disease". Most of the healthcare professionals participating in our study stated that they were not afraid of getting coronavirus before they were infected with the disease. However, almost all of the healthcare professionals stated that they experienced fear during the time they were infected. They experienced the feeling of fear for different reasons.

\section{Sub Theme 1: Fear of the unknown}

Almost all of the participating healthcare professionals stated that they were afraid because they did not know what would happen to them during the time they were infected with coronavirus and what effect this 


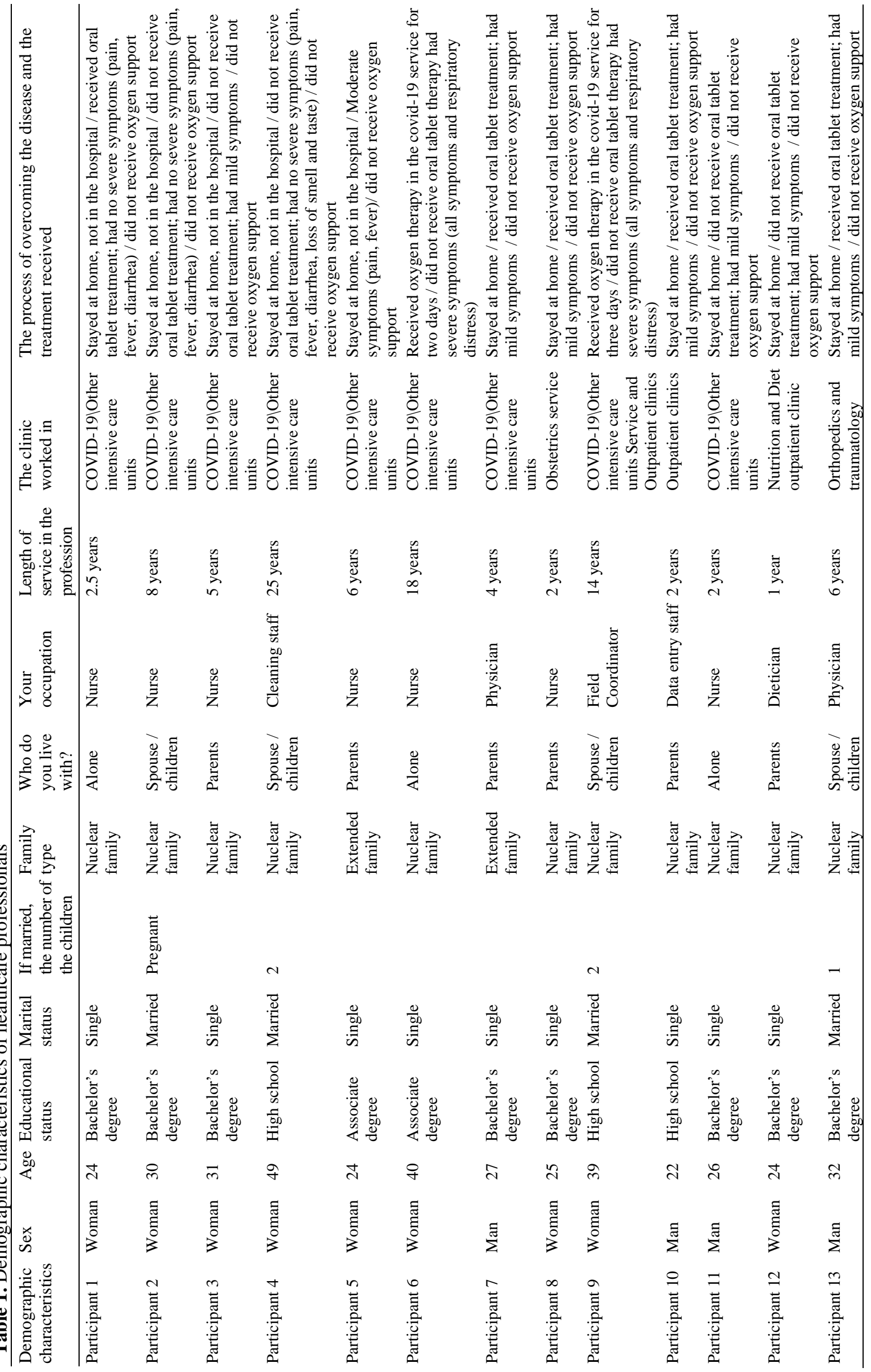


Table 2. Themes and sub-themes produced based on COVID-19 experiences of healthcare professionals

\begin{tabular}{ll}
\hline Themes & Subthemes \\
\hline Fear & Fear of being infected again \\
& Fear of transmitting the disease \\
& Fear of being infected again \\
Social isolation & Unhappiness \\
& Stigma \\
Lack of motivation & Excessive workload \\
& Inadequate number of employees \\
\hline
\end{tabular}

disease would have on them. Although she was young and quite healthy, a nurse stated her experiences as follows:

“... I consoled myself by saying that I am young, it does not infect me, I am a health worker, I am conscious, I am careful. This virus awaits until your slightest mistake and enters your body... We don't know what the virus will do and how it will affect our body. This obscurity still scares me..."(P1).

The biggest fear of a pregnant health worker was the fear of whether the virus would harm her baby. She expressed her fear as follows:

"I was very much frightened because I was pregnant. I wondered if it would give harm to my baby, how much harm it would give. No scientific data on this subject is still clear. This situation worries me too much and I am afraid... I cannot overcome this fear... "(P2).

\section{Sub Theme 2: Fear of transmitting the disease}

Another fear of the participants who were infected was the fear of transmitting the disease to their loved ones and families. The statement of a nurse working in the intensive care unit on this subject was as follows:

"I am not afraid of being infected again, but I am very afraid of infecting my family and loved ones. If they get sick because of me, this hurts me very much. ... Even when I caught the disease, I could not leave my family. Thank goodness they did not become infected... "(P8).

The statements of two nurses working in the COVID-19 service on this issue were as follows:

"I'm not afraid for myself, but I'm afraid when I think of my elderly relatives and humanity. If I infect the elderly in my family and if this causes them to die, then I cannot get rid of this psychology of guilt..."(P11).

"When I learned I was positive, I got very scared and cried sobbingly. I was most afraid of infecting my mother because she already had chronic illnesses. When I found out that my mother was infected, every morning, I woke up in fear, thinking of the possibility that something bad would happen. Would my mother recover? This worry and fear bring about an indescribable feeling of guilt..."(P12).

\section{Sub Theme 3: Fear of being infected again}

Almost all of the healthcare workers who survived the disease once had the fear of being infected again.
Two participants' statements on this issue were as follows:

"... I always have the fear of being infected again. This is because of my pregnancy; my immune system is suppressed and the uncertainty of the next process scares me very much ...” (P2).

"...You have recovered this time, but there is no guarantee that you will not catch COVID-19 again. This is an enormous uncertainty. If I catch it again, I always have a fear of how I will go through it, or if it becomes worse this time..." (P9).

\section{Theme 2. Social isolation}

Most of the participating healthcare professionals stated that they experienced social isolation during and after they had coronavirus, which negatively affected them emotionally. Sub-themes of this theme were "unhappiness" and "stigma".

\section{Sub Theme 1: Unhappiness}

Almost all of the participating healthcare professionals did not enjoy life, were not happy, and felt tired after surviving COVID-19. The statement of a hospital cleaning staff working in the COVID-19 intensive care unit was as follows:

"I survived this illness, but I am still unhappy, weak and tired. My friends used to say go out on the balcony while in quarantine. I didn't want to go out, I even didn't want to talk. Now I don't get any taste from whatever I eat and drink. I'm very unhappy. Staying confined within four walls for 20 days ruined my psychology... "(P4).

The statement of a nurse, COVID-19 field coordinator, is as follows:

"I cried every day during the quarantine. I was so scared of dying, I'm still scared. I was COVID-19 positive twice in a row and cried until the morning. I was completely demoralized and I lost my motivation. My only consolation was my children; I resisted so that they would not be left without a mother. I still have a strange feeling of unhappiness. I want to cry all the time... "(P9).

\section{Sub Theme 2: Stigma}

The vast majority of participating healthcare professionals were stigmatized because they caught COVID-19. The statements of some of the participants who experienced this feeling are as follows:

"I came back home after being diagnosed with COVID-19. I came, but my neighbors treated me as if I had a deadly disease, plague. The way they looked at me and having all eyes on me affected me very badly. I only went to the balcony, even on the balcony I was exposed to strange looks and I was worn out ... "(P2)

"When I first learned that I was positive, I wanted to hide it from people. Because I felt that the way they treated me would change, and that everyone would exclude me. However, when the contact tracing team 
came to the front door, everyone understood I was positive. Nobody asked me if I needed anything. I was constantly told not to leave home, not to step out the door. You don't know what to do in desperation, if you step out the door, the neighbors will immediately inform on you. You feel like you're trapped and you caught the world's worst disease... "(P3).

"I survived COVID-19, but even my very sincere friends are still hesitant to meet me. This makes me very sad. Everyone around me approaches me cautiously, and I feel so excluded... "(P12)

\section{Theme 3. Lack of motivation}

The participants stated that 4-5 people in the service had positive test results at the same time because the healthcare professionals transmitted the disease to each other very quickly, which negatively affected the remaining healthcare professionals and those who caught the disease. Many participants stated that they had to go back to work before they were fully recovered. Of the participants, those who recovered stated that they lacked motivation towards their work after returning to work. The subthemes determined under this theme were "excessive workload" and "inadequate number of employees".

\section{Sub Theme 1. Excessive workload}

The greatest challenge faced by almost all healthcare workers who caught and recovered from Covid-19 when they went back to work was the excessive workload. The constant increase in the number of people infected with COVID-19 increased healthcare professionals' workload. Some of the participants' statements on this issue were as follows:

"This disease is the worst disease I've ever seen. As a healthcare worker, you are tested 10 days after you were COVID-19 positive. If the result is negative, you have to be tested again the next day. If the result is negative again, then you have to go back to work immediately. Nobody cares how you feel, if you are negative, you should go back to work and start working immediately. I am tired, psychologically I do not feel I am ready, I am worn out in this process, I am not ready to work, but I have to... "(P6)

"When I started the work again, nobody said, "You are sick, you have not recovered yet. Let's assign you less work." In fact, my workload doubled. I wanted to get a sick leave, but if I get a sick leave, the workload on the others will increase; I thought it was unfair. I think of myself -I'm weak, unhappy, exhausted, unwilling... "(P4).

\section{Sub Theme 2. Inadequate number of employees}

Of the participants, those who caught COVID-19 and received treatment stated that when they came back to work, very few people were working in the clinic. They stated that during this pandemic, they were very few, trying to do things in the clinics and to provide care to patients. They stated that staff shortage caused lack of motivation. Some of the participants' statements on this issue were as follows:

"Four of my friends in the service I worked with and I were COVID-19 positive, which made it very difficult for the rest of our friends because they had to compensate us. On the other hand, we had to struggle with the disease, and then we immediately went back to work. In this process, there was nothing to encourage us. There was nothing to improve our motivation. There should be something to motivate us morally and materially... "(P13)

"I was positive twice in a row. When I went back to work, I was not healthy at all. I constantly felt dizzy. I was not happy or willing to work at all. I just had to because the number of staff was insufficient. I do not know if I have been helpful to patients, but what else could I do?" (P8).

\section{DISCUSSION}

In the present study, the disease-related experiences of the participating healthcare professionals diagnosed with COVID-19 and recovered were investigated. The main outcomes of the study were fear, social isolation and lack of motivation. Paying attention to healthcare professionals' pandemic-related experiences and concerns is of great importance (Vindrola-Padros et al. 2020). To achieve this goal, it is necessary to understand what the experiences of healthcare professionals are and what they are concerned about. In the present study, it was observed that the feelings commonly defined by the participating healthcare professionals during the period of coronavirus were fear, anxiety and despair. They were afraid of the constantly mutating disease and unpredictable risks, and they were worried for their families. Most of them lacked motivation when they started to work again after they recovered from the disease. In several studies, it has been determined that health workers are afraid of catching the disease in this process (Cao et al. 2020; Chung et al. 2020). In Ahmed et al.'s study, $87 \%$ of healthcare professionals stated that they were afraid of being infected with COVID-19 from a patient or colleague (Ahmed et al. 2020). They felt they had to work. During an epidemic, healthcare professionals work long hours under pressure, often without adequate protective equipment, and accepting all risks (Vindrola-Padros et al. 2020). However, many healthcare workers are at risk of contracting the virus or even of death, and it is almost impossible to reduce this threat to zero (Maunder et al. 2003). This disease, which endangers the lives of healthcare professionals and spreads rapidly, causes them to have extremely hard time (Galehdar et al. 2020). Studies have also revealed that healthcare professionals have longer working hours per week during the pandemic, which can negatively affect their mental health due to the heavy workload it creates (Mo et al. 2020; Sun et al. 2020). On the other 
hand, healthcare professionals with no previous experience in communicable diseases face extra difficulties because they become acquainted with a new work environment in this stressful situation (Q Liu et al. 2020). In this process, they have to both cope with emotional stressors and overcome excessive workload due to the rapidly spreading infection (Adams \& Walls 2020).

Many studies conducted during the coronavirus pandemic have demonstrated that healthcare workers experience many psychological distresses, including depression and anxiety (Allsopp et al. 2019; Amin 2020; Du et al. 2020; Pappa et al. 2020). Moreover, healthcare workers who are infected with the virus and have to cope with the disease are scared more than normal, and feel helpless and unhappy more. In the present study, according to their statements, the participants lacked motivation and they had to work more than usual with fewer people after they recovered from the illness. Due to the shortage of healthcare workers in this process, healthcare workers' right to have annual leave, right to resign for whatever reason, and right to request appointment were banned with the circular letter of the Ministry of Health (Republic of Turkey Ministry of Health 2021). Moreover, the right to an administrative leave given to employees with children under the age of 10, applied throughout Turkey, was denied to health workers (Official Gazette 2021). Most of the healthcare professionals participating in our study also stated that they felt like they were punished because they were banned from accessing their several rights during this pandemic process.

In the present study, according to the statements of the participating healthcare workers, they experienced social isolation, they were unhappy, and they were stigmatized. That coronavirus disease is fatal, is easily transmitted, spreads to many countries in the world in a short time and has created uncertainty about the future and that social interaction has decreased and thus loneliness has increased due to mandatory protective isolation measures have caused health care workers to suffer fear and anxiety (Galehdar et al. 2020; He et al. 2021; Sun et al. 2020; Wang et al. 2020). In studies conducted in previous outbreaks, it was revealed that healthcare workers had the fear of transmitting the disease, worried about the health of their families, experienced isolation and stigma, and were not knowledgeable about the risks of the disease and how these risks would affect them (Imai et al. 2010; Ives et al. 2009; Koh et al. 2011). In a study, it was found that health workers' receiving social support from their teammates, the government and other people in the community protected their mental health (De Kock et al. 2021).

In the present study, almost all the participants experienced fear. Many of them stated that they were afraid of being infected with the disease again and transmitting it to their families. In particular, of the participants, those whose illness was severe or who had relatives whose illness was severe experienced higher levels of fear. If these fears and anxieties are not detected and taken into account, they cause permanent and destructive problems in the long term (Levin 2019). In a study conducted with nurses during the coronavirus pandemic process, what a nurse said actually summarizes all these: 'I'm afraid, because this fear prevents me from communicating with patients well, and I get upset when I cannot communicate well with them. I don't feel good at all as I have to care about people who can infect me at any moment. I may become infected, or I may infect my family, or I may even lose my life...' (Galehdar et al. 2020). Although healthcare professionals think that they are in danger, they still try to fulfill their duties in a quality manner. Healthcare professionals are expected to deliver healthcare services with maximum honesty and courage (Back 2020). During the pandemic, healthcare workers are exposed to heavy workload, lack of resources and protective equipment, and life-threatening risks due to the generally unknown nature of the disease (Maben et al. 2020). Despite all their difficulties, healthcare professionals cannot refuse to fulfill their duties and to serve patients. Therefore, they may experience not only pressures related to professional ethics but also dilemmas of conscience (Torda 2006), which makes them unhappy and hopeless. Our search for studies investigating the stigma experiences of healthcare professionals who recovered from COVID-19 revealed a gap in the literature. In a study, it was stated that healthcare workers who perceived higher degrees of stigma experienced symptoms of COVID-19 and had suicidal thoughts or attempts before or during the COVID-19 pandemic (Teksin et al. 2020). In the same study, it was also determined that healthcare professionals caring for COVID-19 $(+)$ patients felt more stigmatized than individuals not caring for these patients (Teksin et al. 2020).

\section{Limitations of the study}

The present study has several limitations. Although most of the participants in this study are nurses, they are limited to doctors, cleaning personnel, data entry personnel, dietitians, and the result concerns all health professionals, not a single professional group. In the study, health professionals were interviewed once, and repeated interviews were not held. The study data were on the experiences of the health professionals working in a training and research hospital in the city center. The data do not reflect the experiences of health professionals working in small towns regarding contracting COVID-19 disease. In addition, because the participants worked very hard due to the pandemic, there were difficulties in determining the meeting day and time. Therefore, the results obtained from this study are applicable only to the health professionals surveyed and cannot be generalized to all health professionals. Cohort type studies should be conducted to determine the longterm effect of COVID-19 on healthcare professionals. 


\section{CONCLUSIONS AND RECOMMENDATIONS}

At the end of the present study, it was determined that the participating healthcare workers infected with coronavirus experienced serious psychological problems, fear and unhappiness, and social isolation, and that they lacked motivation. The results of the present study indicate that all healthcare professionals infected with coronavirus should be given psychosocial support in line with their needs. Based on the results of the present study, it is recommended that the criteria regarding healthcare professionals' starting work again after they survive the COVID-19 should be reviewed and revised. In particular, healthcare workers who survive illness but cannot fully recover should be evaluated physically and psychologically before going back to work. It is recommended to conduct studies to investigate experiences of healthcare workers who have been diagnosed with COVID-19 and recovered in larger sample groups.

\section{Acknowledgements:}

We would like to thank all the healthcare workers who participated in this study and shared their feelings, opinions, and experiences.

\section{Conflict of interest: None to declare.}

\section{Contribution of individual authors:}

Sadiye Ozcan \& Nurcan Kirca: study design, first draft, approval of the final version, statistical analysis.

\section{References}

1. Adams JG \& Walls RM: Supporting the health care workforce during the covid-19 global epidemic. JAMA 2020; 323:1439-1440

2. Ahmed MA, Jouhar R, Ahmed N, Adnan S, Aftab M, Zafar MS et al: Fear and practice modifications among dentists to combat novel coronavirus disease (COVID-19) outbreak. Int J Environ Res Public Health 2020; 17: 2821

3. Allsopp $K$, Brewin CR, Barrett A, Williams R, Hind D, Chitsabesan $P$ et al: Responding to mental health needs after terror attacks. BMJ 2019; 366:l-4

4. Amin S: The psychology of coronavirus fear: are healthcare professionals suffering from corona-phobia? Int J Healthcare Manag 2020; 13:249-256

5. Back A, Tulsky JA, Arnold RM: Communication skills in the age of COVID-19. Ann Intern Med 2020; 172:759-760

6. Bahar A, Kocak HS, Baglama SS, Cuhadar D: Can psychological resilience protect the mental health of healthcare professionals during the Covid-19 pandemic period? Dubai Med J 2020; 3: 133-139

7. Brooks SK, Gerada C, Chalder T: Review of literature on the mental health of doctors: are specialist services needed? J Ment Health 2011; 20:146-156

8. Cao J, Wei J, Zhu H, Duan Y, Geng W, Hong X et al: A study of basic needs and psychological wellbeing of medical workers in the fever clinic of a tertiary general hospital in Beijing during the COVID-19 Outbreak. Psychother Psychosom 2020; 89: 252-254

9. Chang D, Xu H, Rebaza A, Sharma L, Dela Cruz CS: Protecting health-care workers from subclinical coronavirus infection. Lancet Respir Med 2020; 8: e13

10. Chung JP, Yeung WS: Staff mental health self-assessment during the COVID 19 outbreak East Asian Arch Psychiatry 2020; 30: 34

11. De Kock JH, Latham HA, Leslie SJ, Grindle M, Munoz $S A$, Ellis $L$ et al: A rapid review of the impact of COVID19 on the mental health of healthcare workers: implications for supporting psychological well-being. BMC Public Health 2021; 21: 1-18

12. Du J, Dong L, Wang T, Yuan C, Fu R, Zhang L et al: Psychological symptoms among frontline healthcare workers during COVID-19 outbreak in Wuhan. Gen Hosp Psychiatry 2020; 67: 144-145

13. Edward KL \& Welch T: The extension of Colaizzi's method of phenomenological enquiry. Contemp Nurse 2011; 39: 163-171

14. Galehdar N, Kamran A, Toulabi T, Heydari H: Exploring nurses' experiences of psychological distress during care of patients with COVID-19: a qualitative study. BMC Psychiatry 2020; 20: 489

15. Greenberg N, Docherty M, Gnanapragasam S, Wessely S: Managing mental health challenges faced by healthcare workers during COVID-19 pandemic. BMJ 2020; 368: 1-4

16. Gupta $S$ \& Sahoo S: Pandemic and mental health of the front-line healthcare workers: a review and implications in the Indian context amidst COVID-19. GPSYCH 2020; 33: e100284

17. He Y, Yu R, Ren J: The correlation between psychiatric disorders and COVID-19: a narrative review. Psychiatria Danubina 2021; 33: 76-85

18. Imai H, Matsuishi K, Ito A, Mouri K, Kitamura N, Akimoto $K$ et al: Factors associated with motivation and hesitation to work among health professionals during a public crisis: a cross sectional study of hospital workers in Japan during the pandemic (H1N1) 2009. BMC Public Health 2010; 10:1-8

19. Ives J, Greenfield S, Parry JM, Draper H, Gratus C, Petts $J I$ et al: Healthcare workers' attitudes to working during pandemic influenza: a qualitative study. BMC Public Health 2009; 9: 1-13

20. Jansson M \& Rello J: Mental health in healthcare workers and the covid-19 pandemic era: Novel challenge for critical care. J Intensive \& Crit Care 2020; 6: 1-3

21. Koh Y, Hegney DG \& Drury V: Comprehensive systematic review of healthcare workers' perceptions of risk and use of coping strategies towards emerging respiratory infectious diseases. Int J Evid Based Healthc 2011; 9: 403-419

22. Lai J, Ma S, Wang Y, Cai Z, Hu J, Wei $N$ et al: Factors associated with mental health outcomes among health care workers exposed to coronavirus disease 2019. JAMA 2020; 3: e203976

23. Levin J: Mental health care for survivors and healthcare workers in the aftermath of an outbreak. In: Huremovic D (eds): Psychiatry of Pandemics. 127-141. Springer Nature Switzerland, 2019

24. Liu S, Yang L, Zhang C, Xiang YT, Liu Z, Hu S et al: Online mental health services in China during the COVID-19 outbreak. Lancet Psychiatry 2020; 7: e17-e18. 
25. Liu Q, Luo D, Haase JE, Guo Q, Wang XQ, Liu S et al: The experiences of health-care providers during the COVID-19 crisis in China: a qualitative study. Lancet Glob Health 2020; 8: e790-e798

26. Maben $J \&$ Bridges $J$ : Covid-19: supporting nurses' psychological and mental health. J Clin Nurs 2020; 29: 2742-2750

27. Matua GA \& Van Der Wal DM: Differentiating between descriptive and interpretive phenomenological research approaches. Nurse Res 2015; 22: 22-27

28. Maunder R, Hunter J, Vincent L, Bennett J, Peladeau N, Leszcz $M$ et al: The immediate psychological and occupational impact of the 2003 SARS outbreak in a teaching hospital. CMAJ 2003; 168: 1245-1251

29. Mo Y, Deng L, Zhang L, Lang Q, Liao C, Wang $N$ et al: Work stress among Chinese nurses to support Wuhan for fighting against the COVID-19 epidemic. J Nurs Manage 2020; 28: 1002-1009

30. Nguyen LH, Drew DA, Graham MS, Joshi AD, Guo C, Ma $W$, et al. Risk of COVID-19 among front-line health-care workers and the general community: a prospective cohort study. Lancet Public Health 2020; 5:e475-83. https://doi.org/10.1016/S2468-2667(20)30164-X

31. Official Gazette. (2021, April 14). COVID-19 Kapsaminda Kamu Çalı̧̧anlarına Yönelik Tedbirler. [Measures for Public Employees in the Scope of COVID-19]. Number:31454.

https://www.resmigazete.gov.tr/eskiler/2021/04/202104145.pdf

32. Pappa S, Ntella V, Giannakas T, Giannakoulis VG, Papoutsi E, Katsaounou P: Prevalence of depression, anxiety, and insomnia among healthcare workers during the COVID-19 pandemic: a systematic review and metaanalysis. Brain Behav Immun 2020; 88: 901-907

33. Republic of Turkey Ministry of Health. (2021, August 26). COVID-19 Bilgilendirme Platformu [COVID-19 Information Platform]. https://covid19.saglik.gov.tr/
34. Shanafelt T, Ripp J \& Trockel M: Understanding and addressing sources of anxiety among health care professionals during the covid-19 pandemic. JAMA 2020; 323: 2133-2134

35. Sun $N$, Wei L, Shi S, Jiao D, Song R, Ma L et al: $A$ qualitative study on the psychological experience of caregivers of COVID-19 patients. Am J Infect Control 2020; 48: 592-598.

36. Teksin G, Uluyol OB, Onur OS, Teksin MG, Ozdemir HM: Stigma-related Factors and their Effects on Health-care Workers during COVID-19 Pandemics in Turkey: A Multicenter Study. Med Bull Sisli Etfal Hosp 2020;54: 281-290.

37. Torda A: Ethical issues in pandemic planning. Med J Aust 2006; 185: S73-S76

38. Vindrola-Padros C, Andrews L, Dowrick A, Djellouli N, Fillmore H, Gonzalez E et al: Perceptions and experiences of healthcare workers during the COVID-19 pandemic in the UK. BMJ Open 2020; 10:1-8

39. Wang $S$, Wen X, Dong Y, Liu B \& Cui M: Psychological influence of coronovirus disease 2019 (Covid-19) pandemic on the general public, medical workers, and patients with mental disorders and its countermeasures. Psychosomatics 2020; 61: 616-624

40. World Health Organization (2021, August 26). Coronavirus (COVID-19) Dashboard. https://covid19.who.int/

41. Zhang C, Yang L, Liu S, Ma S, Wang Y, Cai Z et al: Survey of insomnia and related social psychological factors among medical staff involved in the 2019 novel coronavirus disease outbreak. Front Psychiatry 2020; $11: 1-9$

Correspondence:

Assistant Professor Sadiye Ozcan, MD

Yalova University Faculty of Health Sciences, Department of Obstetrics E Gynecological Nursing

Yalova, Turkey

E-mail: sadiyeozcan24@gmail.com 